\title{
Convolution Properties for Certain Classes of Analytic Functions Defined by $q$-Derivative Operator
}

\author{
T. M. Seoudy ${ }^{1,2}$ and M. K. Aouf ${ }^{3}$ \\ ${ }^{1}$ Department of Mathematics, Faculty of Science, Fayoum University, Fayoum 63514, Egypt \\ ${ }^{2}$ The University College at Al-Jamoom, Umm Al-Qura University, Makkah 21421, Saudi Arabia \\ ${ }^{3}$ Department of Mathematics, Faculty of Science, Mansoura University, Mansoura 35516, Egypt \\ Correspondence should be addressed to T. M. Seoudy; tms00@fayoum.edu.eg
}

Received 22 May 2014; Accepted 30 August 2014; Published 14 October 2014

Academic Editor: Juan C. Cortés

Copyright (C) 2014 T. M. Seoudy and M. K. Aouf. This is an open access article distributed under the Creative Commons Attribution License, which permits unrestricted use, distribution, and reproduction in any medium, provided the original work is properly cited.

We investigate convolution properties and coefficients estimates for two classes of analytic functions involving the $q$-derivative operator defined in the open unit disc. Some of our results improve previously known results.

\section{Introduction}

Simply, $h$-calculus or $q$-calculus is ordinary classical calculus without the notion of limits. Here $h$ ostensibly stands for Planck's constant, while $q$ stands for quantum. Recently, the area of $q$-calculus has attracted the serious attention of researchers. This great interest is due to its application in various branches of mathematics and physics. The application of $q$-calculus was initiated by Jackson $[1,2]$. He was the first to develop $q$-integral and $q$-derivative in a systematic way. Later, geometrical interpretation of $q$-analysis has been recognized through studies on quantum groups. It also suggests a relation between integrable systems and $q$-analysis. Aral and Gupta [3-5] defined and studied the $q$-analogue of Baskakov Durrmeyer operator which is based on $q$-analogue of beta function. Another important $q$-generalization of complex operators is $q$-Picard and $q$-Gauss-Weierstrass singular integral operators discussed in [6-8]. Mohammed and Darus [9] studied approximation and geometric properties of these $q$ operators in some subclasses of analytic functions in compact disk. These $q$-operators are defined by using convolution of normalized analytic functions and $q$-hypergeometric functions, where several interesting results are obtained (see also $[10,11])$. A comprehensive study on applications of $q$-calculus in operator theory may be found in [12].
Let $\mathscr{A}$ denote the class of functions of the form:

$$
f(z)=z+\sum_{k=2}^{\infty} a_{k} z^{k}
$$

which are analytic in the open unit disk $\mathbb{U}=\{z \in \mathbb{C}:|z|<1\}$. Let $\mathcal{S}(\alpha)$ and $\mathscr{K}(\alpha) \quad(0 \leq \alpha<1)$ denote the subclasses of $\mathscr{A}$ that consists, respectively, of starlike of order $\alpha$ and convex of order $\alpha$ in $\mathbb{U}$ (see [13]). If $f(z)$ and $g(z)$ are analytic in $\mathbb{U}$, we say that $f(z)$ is subordinate to $g(z)$, written $f(z) \prec g(z)$ if there exists a Schwarz function $\omega$, which (by definition) is analytic in $\mathbb{U}$ with $\omega(0)=0$ and $|\omega(z)|<1$ for all $z \in \mathbb{U}$, such that $f(z)=g(\omega(z)), z \in \mathbb{U}$. Furthermore, if the function $g$ is univalent in $\mathbb{U}$, then we have the following equivalence (see [14-16]):

$$
f(z) \prec g(z) \Longleftrightarrow f(0)=g(0), f(\mathbb{U}) \subset g(\mathbb{U}) \text {. }
$$

For functions $f$ given by (1) and $g$ given by

$$
g(z)=z+\sum_{k=2}^{\infty} b_{k} z^{k}
$$


the Hadamard product or convolution of $f$ and $g$ is defined by

$$
(f * g)(z)=z+\sum_{k=2}^{\infty} a_{k} b_{k} z^{k}=(g * f)(z) .
$$

Let $\mathcal{S}[A, B]$ and $\mathscr{K}[A, B]$ denote the subclasses of the class $\mathscr{A}$ for $-1 \leq B<A \leq 1$ which are defined by (see [17-22])

$$
\begin{gathered}
\mathcal{S}[A, B]=\left\{f \in \mathscr{A}: \frac{z f^{\prime}(z)}{f(z)} \prec \frac{1+A z}{1+B z}, z \in \mathbb{U}\right\}, \\
\mathscr{K}[A, B]=\left\{f \in \mathscr{A}: \frac{\left(z f^{\prime}(z)\right)^{\prime}}{f^{\prime}(z)} \prec \frac{1+A z}{1+B z}, \quad z \in \mathbb{U}\right\} .
\end{gathered}
$$

We note that

$$
\begin{array}{r}
\mathcal{S}[1-2 \alpha,-1]=\mathcal{S}(\alpha), \quad \mathscr{K}[1-2 \alpha,-1]=\mathscr{K}(\alpha) \\
(0 \leq \alpha<1) .
\end{array}
$$

For function $f \in \mathscr{A}$ given by (1) and $0<q<1$, the $q$ derivative of a function $f$ is defined by (see [1])

$$
D_{q} f(z)=\frac{f(q z)-f(z)}{(q-1) z} \quad(z \neq 0),
$$

and $D_{q} f(0)=f^{\prime}(0)$. From (7), we deduce that

$$
D_{q} f(z)=1+\sum_{k=2}^{\infty}[k]_{q} a_{k} z^{k-1}, \quad z \neq 0
$$

where

$$
[k]_{q}=\frac{1-q^{k}}{1-q} .
$$

As $q \rightarrow 1,[k]_{q} \rightarrow k$. For a function $h(z)=z^{k}$, we observe that

$$
\begin{aligned}
& D_{q} h(z)=D_{q}\left(z^{k}\right)=\frac{1-q^{k}}{1-q} z^{k-1}=[k]_{q} z^{k-1}, \\
& \lim _{q \rightarrow 1} D_{q} h(z)=\lim _{q \rightarrow 1}[k]_{q} z^{k-1}=k z^{k-1}=h^{\prime}(z),
\end{aligned}
$$

where $h^{\prime}$ is the ordinary derivative.

Making use of the $q$-derivative $D_{q} f(z)$, we introduce the subclasses $\mathcal{S}_{q}[A, B]$ and $\mathscr{K}_{q}[A, B]$ of $\mathscr{A}$ for $0<q<1$ and $-1 \leq B<A \leq 1$ as follows:

$$
\begin{aligned}
& \mathcal{S}_{q}[A, B]=\left\{f \in \mathscr{A}: \frac{z D_{q} f(z)}{f(z)} \prec \frac{1+A z}{1+B z}, z \in \mathbb{U}\right\}, \\
& \mathscr{K}_{q}[A, B] \\
& \quad=\left\{f \in \mathscr{A}: \frac{D_{q}\left(z D_{q} f(z)\right)}{D_{q} f(z)} \prec \frac{1+A z}{1+B z}, z \in \mathbb{U}\right\} .
\end{aligned}
$$

We note that

$$
\begin{aligned}
& \text { (i) } \mathcal{S}_{q}[1-2 \alpha,-1]=\mathcal{S}_{q}(\alpha)(0 \leq \alpha<1) \\
& \mathcal{S}_{q}(\alpha)=\left\{f \in \mathscr{A}: \operatorname{Re} \frac{z D_{q} f(z)}{f(z)}>\alpha, z \in \mathbb{U}\right\} ;
\end{aligned}
$$

(ii) $\mathscr{K}_{q}[1-2 \alpha,-1]=\mathscr{K}_{q}(\alpha) \quad(0 \leq \alpha<1)$

$$
\mathscr{K}_{q}(\alpha)=\left\{f \in \mathscr{A}: \operatorname{Re} \frac{D_{q}\left(z D_{q} f(z)\right)}{D_{q} f(z)}>\alpha, z \in \mathbb{U}\right\}
$$

(iii) $\mathcal{S}_{q}[(1-2 \alpha) \beta,-\beta]=\mathcal{S}_{q}(\alpha, \beta) \quad(0 \leq \alpha<1,0<\beta \leq 1)$ $\mathcal{S}_{q}(\alpha, \beta)$

$$
=\left\{f \in \mathscr{A}:\left|\frac{\left(z D_{q} f(z) / f(z)\right)-1}{\left(z D_{q} f(z) / f(z)\right)+1-2 \alpha}\right|<\beta, z \in \mathbb{U}\right\},
$$

(iv) $\mathscr{K}_{q}[(1-2 \alpha) \beta,-\beta]=\mathscr{K}_{q}(\alpha, \beta) \quad(0 \leq \alpha<1,0<\beta \leq$ 1)

$$
\begin{gathered}
\mathscr{K}_{q}(\alpha, \beta) \\
=\left\{f \in \mathscr{A}:\left|\frac{\left(D_{q}\left(z D_{q} f(z)\right) / D_{q} f(z)\right)-1}{\left(D_{q}\left(z D_{q} f(z)\right) / D_{q} f(z)\right)+1-2 \alpha}\right|<\beta,\right. \\
z \in \mathbb{U}\},
\end{gathered}
$$

(v)

$$
\begin{aligned}
\lim _{q \rightarrow 1} \mathcal{S}_{q}[A, B] & =\left\{f \in \mathscr{A}: \lim _{q \rightarrow 1} \frac{z D_{q} f(z)}{f(z)} \prec \frac{1+A z}{1+B z}\right\} \\
= & \mathcal{S}[A, B], \\
\lim _{q \rightarrow 1} \mathscr{K}_{q}[A, B] & \\
& =\left\{f \in \mathscr{A}: \lim _{q \rightarrow 1} \frac{D_{q}\left(z D_{q} f(z)\right)}{D_{q} f(z)} \prec \frac{1+A z}{1+B z}\right\} \\
& =\mathscr{K}[A, B] .
\end{aligned}
$$

From (11), we have

$$
f \in \mathscr{K}_{q}[A, B] \Longleftrightarrow z D_{q} f \in \mathcal{S}_{q}[A, B] .
$$

In this paper, we investigate convolution properties, the necessary and sufficient condition and coefficient estimates for the classes $\mathcal{S}_{q}[A, B]$ and $\mathscr{K}_{q}[A, B]$ associated with the $q$ derivative $D_{q} f(z)$. The motivation of this paper is to improve and generalize previously known results. 


\section{Convolution Properties}

Unless otherwise mentioned, we assume throughout this section that $\theta \in[0,2 \pi), 0<q<1$ and $-1 \leq B<A \leq 1$.

Theorem 1. The function $f$ defined by (1) is in the class $\mathcal{S}_{q}[A, B]$ if and only if

$$
\frac{1}{z}\left[f(z) * \frac{z-L q z^{2}}{(1-z)(1-q z)}\right] \neq 0 \quad(z \in \mathbb{U})
$$

for all $L=L_{\theta}=\left(e^{-i \theta}+A\right) /(A-B)$ and also $L=1$.

Proof. First suppose $f$ defined by (1) is in the class $\mathcal{S}_{q}[A, B]$; we have

$$
\frac{z D_{q} f(z)}{f(z)} \prec \frac{1+A z}{1+B z} .
$$

Since the function from the left-hand side of the subordination is analytic in $\mathbb{U}$, it follows $f(z) \neq 0, z \in \mathbb{U}^{*}=\mathbb{U} \backslash\{0\}$; that is, $(1 / z) f(z) \neq 0, z \in \mathbb{U}$, and this is equivalent to the fact that (18) holds for $L=1$. From (19) according to the subordination of two analytic functions we say that there exists a function $w(z)$ analytic in $\mathbb{U}$ with $w(0)=0,|w(z)|<1$ such that

$$
\frac{z D_{q} f(z)}{f(z)}=\frac{1+A w(z)}{1+B w(z)} \quad(z \in \mathbb{U})
$$

which is equivalent to

$$
\frac{z D_{q} f(z)}{f(z)} \neq \frac{1+A e^{i \theta}}{1+B e^{i \theta}} \quad(z \in \mathbb{U} ; 0 \leq \theta<2 \pi),
$$

or

$$
\begin{array}{r}
\frac{1}{z}\left[\left(1+B e^{i \theta}\right) z D_{q} f(z)-\left(1+A e^{i \theta}\right) f(z)\right] \neq 0 \\
(z \in \mathbb{U} ; 0 \leq \theta<2 \pi) .
\end{array}
$$

Since

$$
\begin{gathered}
f(z) * \frac{z}{1-z}=f(z), \\
f(z) * \frac{z}{(1-z)(1-q z)}=z D_{q} f(z) .
\end{gathered}
$$

Now from (23), we may write (22) as

$$
\begin{aligned}
& \frac{1}{z}\left[f(z) *\left(\frac{\left(1+B e^{i \theta}\right) z}{(1-z)(1-q z)}-\frac{\left(1+A e^{i \theta}\right) z}{1-z}\right)\right] \\
& =\frac{(B-A) e^{i \theta}}{z} \\
& \quad \times\left[f(z) * \frac{z-\left(\left(e^{-i \theta}+A\right) /(A-B)\right) q z^{2}}{(1-z)(1-q z)}\right] \neq 0 \\
& (z \in \mathbb{U} ; 0 \leq \theta<2 \pi),
\end{aligned}
$$

which leads to (18), which proves the necessary part of Theorem 1.
Reversely, because assumption (18) holds for $L=1$, it follows that $(1 / z) f(z) \neq 0$ for all $z \in \mathbb{U}$; hence, the function $\varphi(z)=z D_{q} f(z) / f(z)$ is analytic in $\mathbb{U}$ (i.e., it is regular at $z_{0}=0$, with $\left.\varphi(0)=0\right)$. Since it was shown in the first part of the proof that assumption (18) is equivalent to (21), we obtain that

$$
\frac{z D_{q} f(z)}{f(z)} \neq \frac{1+A e^{i \theta}}{1+B e^{i \theta}} \quad(z \in \mathbb{U} ; 0 \leq \theta<2 \pi),
$$

and if we denote

$$
\psi(z)=\frac{1+A z}{1+B z} \quad(z \in \mathbb{U}),
$$

relation (25) shows that $\varphi(\mathbb{U}) \cap \psi(\mathbb{U})=\emptyset$. Thus, the simply connected domain $\varphi(\mathbb{U})$ is included in a connected component of $\mathbb{C} \backslash \psi(\partial \mathbb{U})$. From here, using the fact that $\varphi(0)=\psi(0)$ together with the univalence of the function $\psi$, it follows that $\varphi(z) \prec \psi(z)$, which represents in fact subordination (19); that is, $f \in \mathcal{S}_{q}[A, B]$. This completes the proof of Theorem 1 .

Taking $q \rightarrow 1^{-}$in Theorem 1 , we obtain the following result which improves the convolution result of Aouf and Seoudy [23, Theorem 1] and also the result of Silverman and Silvia [21, Theorem 7].

Corollary 2. The function $f$ defined by (1) is in the class $\mathcal{S}[A, B]$ if and only if

$$
\frac{1}{z}\left[f(z) * \frac{z-L z^{2}}{(1-z)^{2}}\right] \neq 0 \quad(z \in \mathbb{U})
$$

for all $L=L_{\theta}=\left(e^{-i \theta}+A\right) /(A-B)$ and also $L=1$.

Putting $A=1-2 \alpha(0 \leq \alpha<1)$ and $B=-1$ in Theorem 1, we obtain the following corollary.

Corollary 3. The function $f$ defined by (1) is in the class $\mathcal{S}_{q}(\alpha)(0 \leq \alpha<1)$ if and only if

$$
\frac{1}{z}\left[f(z) * \frac{z-M q z^{2}}{(1-z)(1-q z)}\right] \neq 0 \quad(z \in \mathbb{U})
$$

for all $M=M_{\theta}=\left(e^{-i \theta}+1-2 \alpha\right) / 2(1-\alpha), 0 \leq \alpha<1$, and also $M=1$.

Taking $q \rightarrow 1^{-}$in Corollary 3, we obtain the following result which improves the convolution result of Silverman et al. [22, Theorems 1].

Corollary 4. The function $f$ defined by (1) is in the class $\mathcal{S}(\alpha)(0 \leq \alpha<1)$ if and only if

$$
\frac{1}{z}\left[f(z) * \frac{z-M z^{2}}{(1-z)^{2}}\right] \neq 0 \quad(z \in \mathbb{U})
$$

for all $M=M_{\theta}=\left(e^{-i \theta}+1-2 \alpha\right) / 2(1-\alpha), 0 \leq \alpha<1$, and also $M=1$. 
Theorem 5. The function $f$ defined by (1) is in the class $\mathscr{K}_{q}[A, B]$ if and only if

$$
\frac{1}{z}\left[f(z) * \frac{z+[1-(q+1) L] q z^{2}}{(1-z)(1-q z)\left(1-q^{2} z\right)}\right] \neq 0 \quad(z \in \mathbb{U})
$$

for all $L=L_{\theta}=\left(e^{-i \theta}+A\right) /(A-B)$ and also $L=1$.

Proof. Set

$$
g(z)=\frac{z-L q z^{2}}{(1-z)(1-q z)}
$$

and we note that

$$
z D_{q} g(z)=\frac{z+[1-(q+1) L] q z^{2}}{(1-z)(1-q z)\left(1-q^{2} z\right)} .
$$

From the identity $z D_{q} f(z) * g(z)=f(z) * z D_{q} g(z) \quad(f, g \in$ $\mathscr{A})$ and the fact that

$$
f \in \mathscr{K}_{q}[A, B] \Longleftrightarrow z D_{q} f(z) \in \mathcal{S}_{q}[A, B]
$$

the result follows from Theorem 1 .

Taking $q \rightarrow 1^{-}$in Theorem 1 , we obtain the following result which improves the result of Aouf and Seoudy [23, Theorem 2].

Corollary 6. The function $f$ defined by (1) is in the class $\mathscr{K}[A, B]$ if and only if

$$
\frac{1}{z}\left[f(z) * \frac{z+[1-2 L] z^{2}}{(1-z)^{3}}\right] \neq 0 \quad(z \in \mathbb{U})
$$

for all $L=L_{\theta}=\left(e^{-i \theta}+A\right) /(A-B)$ and also $L=1$.

Putting $A=1-2 \alpha(0 \leq \alpha<1)$ and $B=-1$ in Theorem 5, we obtain the following corollary.

Corollary 7. The function $f$ defined by (1) is in the class $\mathscr{K}_{q}(\alpha)(0 \leq \alpha<1)$ if and only if

$$
\frac{1}{z}\left[f(z) * \frac{z+[1-(q+1) L] q z^{2}}{(1-z)(1-q z)\left(1-q^{2} z\right)}\right] \neq 0 \quad(z \in \mathbb{U})
$$

for all $M=M_{\theta}=\left(e^{-i \theta}+1-2 \alpha\right) / 2(1-\alpha), 0 \leq \alpha<1$, and also $L=1$.

Taking $q \rightarrow 1^{-}$in Corollary 7 , we obtain the following result which improves the convolution result of Silverman et al. [22, Theorem 2].

Corollary 8. The function $f$ defined by (1) is in the class $\mathscr{K}(\alpha)(0 \leq \alpha<1)$ if and only if

$$
\frac{1}{z}\left[f(z) * \frac{z+[1-2 L] q z^{2}}{(1-z)^{3}}\right] \neq 0 \quad(z \in \mathbb{U})
$$

for all $M=M_{\theta}=\left(e^{-i \theta}+1-2 \alpha\right) / 2(1-\alpha), 0 \leq \alpha<1$, and also $L=1$.
Theorem 9. A necessary and sufficient condition for the function $f$ defined by (1) to be in the class $\mathcal{S}_{q}[A, B]$ is that

$$
1-\sum_{k=2}^{\infty} \frac{[k]_{q}\left(e^{-i \theta}+B\right)-e^{-i \theta}-A}{A-B} a_{k} z^{k-1} \neq 0
$$

$(z \in \mathbb{U})$

Proof. From Theorem 1, we find that $f \in \mathcal{S}_{q}[A, B]$ if and only if

$$
\frac{1}{z}\left[f(z) * \frac{z-L q z^{2}}{(1-z)(1-q z)}\right] \neq 0 \quad(z \in \mathbb{U})
$$

for all $L=L_{\theta}=\left(e^{-i \theta}+A\right) /(A-B)$ and also for $L=1$. The left-hand side of (38) can be written as

$$
\begin{aligned}
\frac{1}{z}[f & \left.(z) *\left(\frac{z}{(1-z)(1-q z)}-\frac{L q z^{2}}{(1-z)(1-q z)}\right)\right] \\
& =\frac{1}{z}\left\{z D_{q} f(z)-L\left[z D_{q} f(z)-f(z)\right]\right\} \\
& =1-\sum_{k=2}^{\infty}\left([k]_{q}(L-1)-L\right) a_{k} z^{k-1} .
\end{aligned}
$$

Thus, the proof of The Theorem 9 is completed. result.

Taking $q \rightarrow 1^{-}$in Theorem 9 , we obtain the following

Corollary 10. A necessary and sufficient condition for the function $f$ defined by (1) to be in the class $\mathcal{S}[A, B]$ is that

$$
1-\sum_{k=2}^{\infty} \frac{k\left(e^{-i \theta}+B\right)-e^{-i \theta}-A}{A-B} a_{k} z^{k-1} \neq 0 \quad(z \in \mathbb{U}) .
$$

Putting $A=1-2 \alpha(0 \leq \alpha<1)$ and $B=-1$ in Theorem 9, we obtain the following corollary.

Corollary 11. A necessary and sufficient condition for the function $f$ defined by (1) to be in the class $\mathcal{S}_{q}(\alpha)$ is that

$$
1-\sum_{k=2}^{\infty} \frac{[k]_{q}\left(e^{-i \theta}-1\right)-e^{-i \theta}-1+2 \alpha}{2(1-\alpha)} a_{k} z^{k-1} \neq 0 \quad(z \in \mathbb{U}) .
$$

Taking $q \rightarrow 1^{-}$in Corollary 11, we obtain the following corollary which improves the result of Ahuja [17, Corollary 1 when $n=0$ ].

Corollary 12. A necessary and sufficient condition for the function $f$ defined by (1) to be in the class $\mathcal{S}(\alpha)$ is that

$$
1-\sum_{k=2}^{\infty} \frac{k\left(e^{-i \theta}-1\right)-e^{-i \theta}-1+2 \alpha}{2(1-\alpha)} a_{k} z^{k-1} \neq 0 \quad(z \in \mathbb{U}) .
$$


Theorem 13. A necessary and sufficient condition for the function $f(z)$ defined by (1) to be in the class $\mathscr{K}_{q}[A, B]$ is that

$$
1-\sum_{k=2}^{\infty}[k]_{q} \frac{[k]_{q}\left(e^{-i \theta}+B\right)-e^{-i \theta}-A}{A-B} a_{k} z^{k-1} \neq 0 \quad(z \in \mathbb{U}) .
$$

Proof. From Theorem 5, we find that $f \in \mathscr{K}_{q}[A, B]$ if and only if

$$
\frac{1}{z}\left\{f(z) * \frac{z+[1-(q+1) L] q z^{2}}{(1-z)(1-q z)\left(1-q^{2} z\right)}\right\} \neq 0 \quad(z \in \mathbb{U}),
$$

for all $L=L_{\theta}=\left(e^{-i \theta}+A\right) /(A-B)$ and also for $L=1$. The left-hand side of (44) may be written as

$$
\begin{gathered}
\frac{1}{z}\left\{f ( z ) * \left(\frac{z}{(1-z)(1-q z)\left(1-q^{2} z\right)}\right.\right. \\
\left.\left.\quad+\frac{[1-(q+1) L] q z^{2}}{(1-z)(1-q z)\left(1-q^{2} z\right)}\right)\right\} \\
=\frac{1}{z}\left\{q z^{2} D_{q}\left(D_{q} f(z)\right)+z D_{q} f(z)\right. \\
\left.-L\left[q z^{2} D_{q}\left(D_{q} f(z)\right)\right]\right\} \\
=1-\sum_{k=2}^{\infty}[k]_{q} \frac{[k-1]_{q} q e^{-i \theta}-A+[k]_{q} B}{A-B} a_{k} z^{k-1},
\end{gathered}
$$

and this proves Theorem 13 . result.

Taking $q \rightarrow 1^{-}$in Theorem 13 , we obtain the following

Corollary 14. A necessary and sufficient condition for the function $f(z)$ defined by (1) to be in the class $\mathscr{K}[A, B]$ is that

$$
1-\sum_{k=2}^{\infty} k \frac{k\left(e^{-i \theta}+B\right)-e^{-i \theta}-A}{A-B} a_{k} z^{k-1} \neq 0 \quad(z \in \mathbb{U}) .
$$

Putting $A=1-2 \alpha(0 \leq \alpha<1)$ and $B=-1$ in Theorem 13, we obtain the following corollary.

Corollary 15. A necessary and sufficient condition for the function $f$ defined by (1) to be in the class $\mathscr{K}_{q}(\alpha)(0 \leq \alpha<1)$ is that

$$
1-\sum_{k=2}^{\infty}[k]_{q} \frac{[k]_{q}\left(e^{-i \theta}-1\right)-e^{-i \theta}-1+2 \alpha}{2(1-\alpha)} a_{k} z^{k-1} \neq 0
$$

$$
(z \in \mathbb{U}) \text {. }
$$

Taking $q \rightarrow 1^{-}$in Corollary 15, we obtain the following corollary which improves the result of Ahuja [17, Corollary 1 when $n=1]$.
Corollary 16. A necessary and sufficient condition for the function $f$ defined by (1) to be in the class $\mathscr{K}(\alpha)(0 \leq \alpha<1)$ is that

$$
1-\sum_{k=2}^{\infty} k \frac{k\left(e^{-i \theta}-1\right)-e^{-i \theta}-1+2 \alpha}{2(1-\alpha)} a_{k} z^{k-1} \neq 0 \quad(z \in \mathbb{U}) .
$$

\section{Coefficient Estimates}

As an application of Theorems 9 and 13, we next determine coefficient estimate and inclusion property for a function of form (1) to be in the classes $\mathcal{S}_{q}[A, B]$ and $\mathscr{K}_{q}[A, B]$.

Theorem 17. If the function $f$ defined by (1) satisfies the following inequality:

$$
\sum_{k=2}^{\infty}\left\{[k]_{q}(1-B)-1+A\right\}\left|a_{k}\right| \leq A-B
$$

then $f \in \mathcal{S}_{q}[A, B]$.

Proof. Since

$$
\begin{aligned}
& \left|1-\sum_{k=2}^{\infty} \frac{[k]_{q}\left(e^{-i \theta}+B\right)-e^{-i \theta}-A}{A-B} a_{k} z^{k-1}\right| \\
& \quad>1-\sum_{k=2}^{\infty}\left|\frac{[k]_{q}\left(e^{-i \theta}+B\right)-e^{-i \theta}-A}{A-B}\right|\left|a_{k}\right| \\
& \quad=1-\sum_{k=2}^{\infty} \frac{\left|[k]_{q}\left(e^{-i \theta}+B\right)-e^{-i \theta}-A\right|}{A-B}\left|a_{k}\right| \\
& \quad>1-\sum_{k=2}^{\infty} \frac{[k]_{q}(1-B)-1+A}{A-B}\left|a_{k}\right|>0
\end{aligned}
$$

the result follows from Theorem 9.

Taking $q \rightarrow 1^{-}$in Theorem 17 , we obtain the result of Ahuja [17, Theorem 3 when $n=0]$.

Corollary 18. If the function $f$ defined by (1) satisfies the following inequality:

$$
\sum_{k=2}^{\infty}[k(1-B)-1+A]\left|a_{k}\right| \leq A-B
$$

then $f \in \mathcal{S}[A, B]$.

Putting $A=1-2 \alpha(0 \leq \alpha<1)$ and $B=-1$ in Theorem 21, we obtain the following corollary.

Corollary 19. If the function $f$ defined by (1) satisfies the following inequality:

$$
\sum_{k=2}^{\infty}\left([k]_{q}-\alpha\right)\left|a_{k}\right| \leq 1-\alpha,
$$

then $f \in \mathcal{S}_{q}(\alpha)$. 
Taking $q \rightarrow 1^{-}$in Corollary 19, we obtain the following corollary obtained by Silverman [24].

Corollary 20. If the function $f$ defined by (1) satisfies the following inequality:

$$
\sum_{k=2}^{\infty}(k-\alpha)\left|a_{k}\right| \leq 1-\alpha,
$$

then $f \in \mathcal{S}(\alpha)$.

Similarly, we can prove the following theorem.

Theorem 21. If the function $f$ defined by (1) satisfies the following inequality:

$$
\sum_{k=2}^{\infty}[k]_{q}\left\{[k]_{q}(1-B)-1+A\right\}\left|a_{k}\right| \leq A-B,
$$

then $f \in \mathscr{K}_{q}[A, B]$.

Taking $q \rightarrow 1^{-}$in Theorem 21 , we obtain the result of Ahuja [17, Theorem 3 when $n=1]$.

Corollary 22. If the function $f$ defined by (1) satisfies the following inequality:

$$
\sum_{k=2}^{\infty} k[k(1-B)-1+A]\left|a_{k}\right| \leq A-B,
$$

then $f \in \mathscr{K}[A, B]$.

Putting $A=1-2 \alpha(0 \leq \alpha<1)$ and $B=-1$ in Theorem 21, we obtain the following corollary.

Corollary 23. The function $f$ defined by (1) belongs to the class $\mathscr{K}_{q}(\alpha)(0 \leq \alpha<1)$ if

$$
\sum_{k=2}^{\infty}[k]_{q}\left([k]_{q}-\alpha\right)\left|a_{k}\right| \leq 1-\alpha .
$$

Taking $q \rightarrow 1^{-}$in Corollary 23, we obtain the following corollary obtained by Silverman [24].

Corollary 24. The function $f$ defined by (1) belongs to the class $\mathscr{K}(\alpha)(0 \leq \alpha<1)$ if

$$
\sum_{k=2}^{\infty} k(k-\alpha)\left|a_{k}\right| \leq 1-\alpha
$$

\section{Conflict of Interests}

The authors declare that there is no conflict of interests regarding the publication of this paper.

\section{Acknowledgment}

The authors are grateful to the referees for their valuable suggestions.

\section{References}

[1] F. H. Jackson, "On $q$-functions and a certain difference operator," Transactions of the Royal Society of Edinburgh, vol. 46, no. 2, pp. 253-281, 1909.

[2] F. H. Jackson, “On $q$-definite integrals," The Quarterly Journal of Pure and Applied Mathematics, vol. 41, pp. 193-203, 1910.

[3] A. Aral and V. Gupta, "On q-Baskakov type operators," Demonstratio Mathematica, vol. 42, no. 1, pp. 109-122, 2009.

[4] A. Aral and V. Gupta, "On the Durrmeyer type modification of the q-Baskakov type operators," Nonlinear Analysis: Theory, Methods and Applications, vol. 72, no. 3-4, pp. 1171-1180, 2010.

[5] A. Aral and V. Gupta, "Generalized q-Baskakov operators," Mathematica Slovaca, vol. 61, no. 4, pp. 619-634, 2011.

[6] G. A. Anastassiou and S. G. Gal, "Geometric and approximation properties of some singular integrals in the unit disk," Journal of Inequalities and Applications, vol. 2006, Article ID 17231, 19 pages, 2006.

[7] G. A. Anastassiou and S. G. Gal, "Geometric and approximation properties of generalized singular integrals in the unit disk," Journal of the Korean Mathematical Society, vol. 43, no. 2, pp. 425-443, 2006.

[8] A. Aral, "On the generalized Picard and Gauss Weierstrass singular integrals," Journal of Computational Analysis and Applications, vol. 8, no. 3, pp. 249-261, 2006.

[9] A. Mohammed and M. Darus, "A generalized operator involving the $q$-hypergeometric function," Matematichki Vesnik, vol. 65, no. 4, pp. 454-465, 2013.

[10] H. Al Dweby and M. Darus, "On harmonic meromorphic functions associated with basic hypergeometric functions," The Scientific World Journal, vol. 2013, Article ID 164287, 7 pages, 2013.

[11] H. Aldweby and M. Darus, "A subclass of harmonic univalent functions associated with $q$-analogue of Dziok-Srivastava operator," ISRN Mathematical Analysis, vol. 2013, Article ID 382312, 6 pages, 2013.

[12] A. Aral, V. Gupta, and R. P. Agarwal, Applications of q-Calculus in Operator Theory, Springer, New York, NY, USA, 2013.

[13] G. Murugusundaramoorthy and N. Magesh, "Starlike and convex functions of complex order involving the Dziok-Srivastava operator," Integral Transforms and Special Functions, vol. 18, no. 5-6, pp. 419-425, 2007.

[14] T. Bulboacă, Differential Subordinations and Superordinations, Recent Results, House of Scientific Book Publishers, ClujNapoca, Romania, 2005.

[15] S. S. Miller and P. T. Mocanu, Differential Subordination: Theory and Applications, vol. 225 of Monographs and Textbooks in Pure and Applied Mathematics, Marcel Dekker, New York, NY, USA, 2000.

[16] S. S. Miller and P. T. Mocanu, "Subordinants of differential superordinations," Complex Variables, vol. 48, no. 10, pp. 815826, 2003.

[17] O. P. Ahuja, "Families of analytic functions related to Ruscheweyh derivatives and subordinate to convex functions," Yokohama Mathematical Journal, vol. 41, no. 1, pp. 39-50, 1993.

[18] R. M. Goel and B. S. Mehrok, "On the coefficients of a subclass of starlike functions," Indian Journal of Pure and Applied Mathematics, vol. 12, no. 5, pp. 634-647, 1981.

[19] W. Janowski, "Some extremal problems for certain families of analytic functions," Bulletin of the Polish Academy of Sciences, vol. 21, pp. 17-25, 1973. 
[20] W. Janowski, "Some extremal problems for certain families of analytic functions," Annales Polonici Mathematici, vol. 28, pp. 297-326, 1973.

[21] H. Silverman and E. M. Silvia, "Subclasses of starlike functions subordinate to convex functions," Canadian Journal of Mathematics, vol. 1, pp. 48-61, 1985.

[22] H. Silverman, E. M. Silvia, and D. Telage, "Convolution conditions for convexity starlikeness and spiral-likeness," Mathematische Zeitschrift, vol. 162, no. 2, pp. 125-130, 1978.

[23] M. K. Aouf and T. M. Seoudy, "Classes of analytic functions related to the Dziok-Srivastava operator," Integral Transforms and Special Functions, vol. 22, no. 6, pp. 423-430, 2011.

[24] H. Silverman, "Univalent functions with negative coefficients," Proceedings of the American Mathematical Society, vol. 51, pp. 109-116, 1975. 


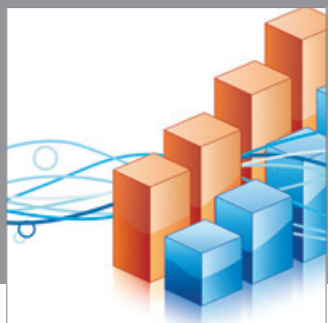

Advances in

Operations Research

mansans

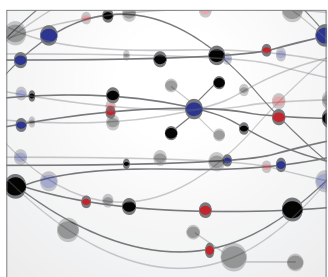

The Scientific World Journal

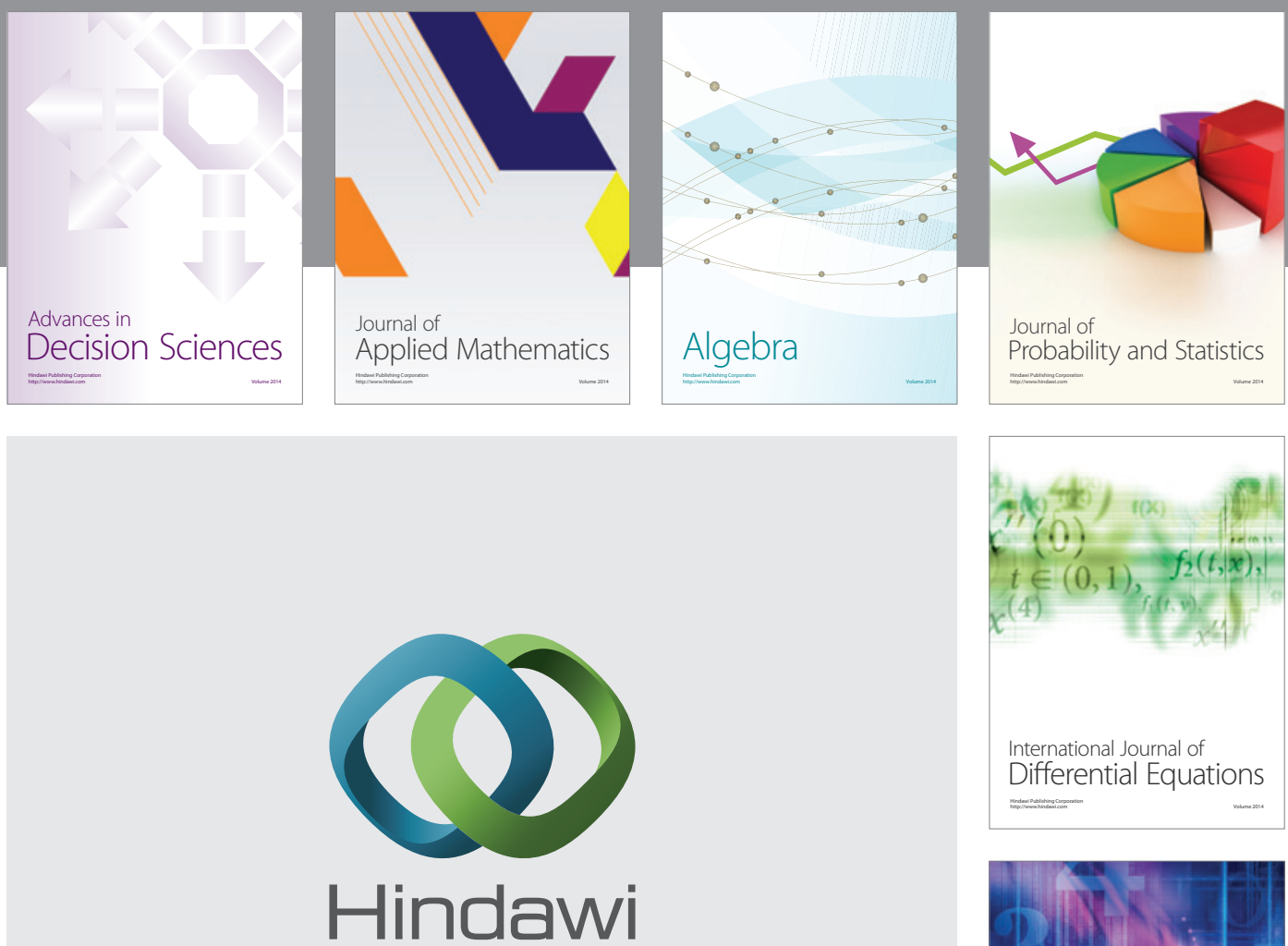

Submit your manuscripts at http://www.hindawi.com
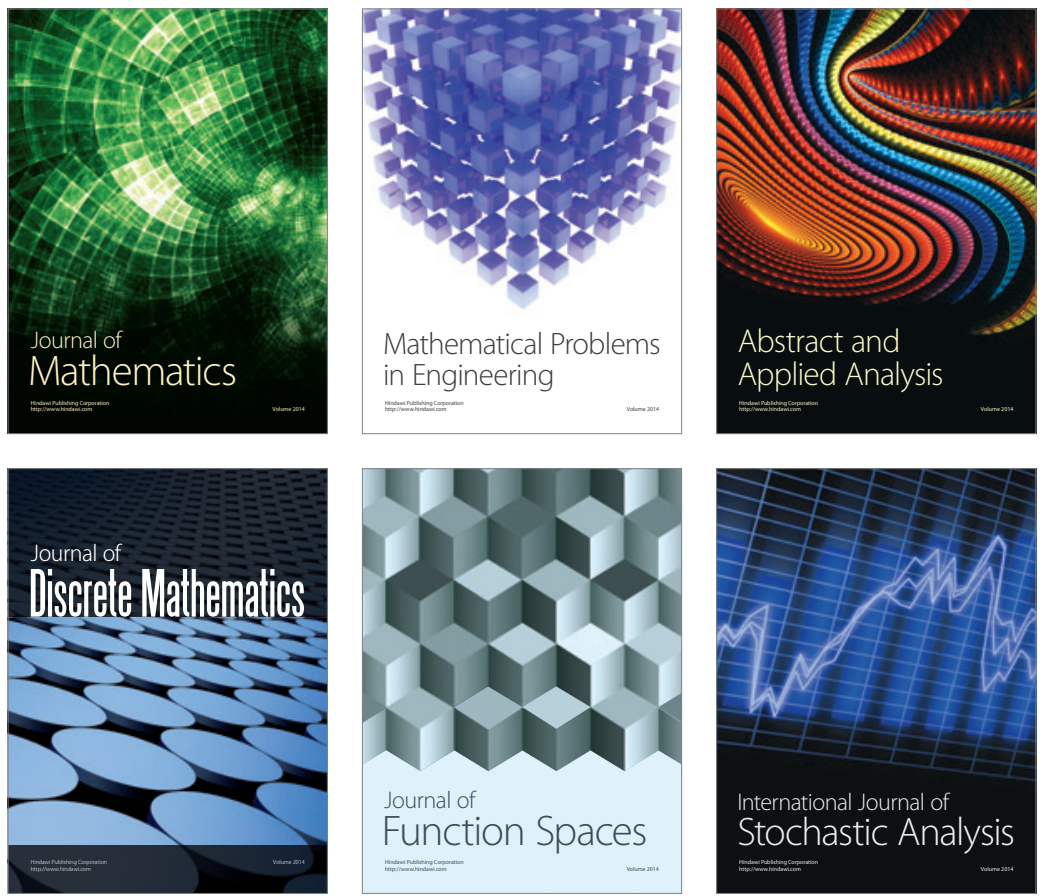

Journal of

Function Spaces



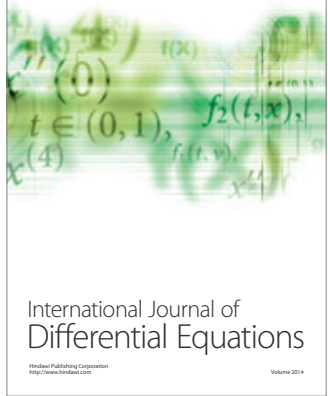
